\title{
Creating Anti-HIV-infection effect by synthesis of aunps from rosa canina I. fruit extract
}

\begin{abstract}
Background and objective: Rosa canina fruit extract has evident immunomodulatory properties. The objective of this study was to produce AuNPs using this extract and to compare their anti-HIV effects with AuNPs produced through the conventional Turkevich method.

Method: In this study, AuNPs were produced through Turkevich method and through reduction using Rosa canina fruit extract. They were characterized through TEM, DLS, UV-visible and FT-IR analyses and their cytotoxicity was evaluated through MTT assay. After HIVs were separated from PBMCs of the HIV-infected human body, they were exposed to interaction with both types of nanoparticles. p24 antigen test was utilized to obtain and compare the percent inhibition of p24 antigen, as a symbol of anti-HIV activity of each type.
\end{abstract}

Results: In contrast with AuNPs produced through the conventional Turkevich method, nanoparticles produced with the extract had better size stability and preserved their average size of $27.7 \mathrm{~nm}$ in physiological condition. For nanoparticles produced with the extract, when the viruses interacted with the nanoparticles and then infected the healthy PBMCs, the percent inhibition of p 24 antigen was $61 \%$; but when the PBMCs were infected by the viruses and then exposed to these nanoparticles, the percent inhibition was $53 \%$.

Conclusion: Rosa canina fruit extract can act as a novel and potent reducing and stabilizing agent for the synthesis of AuNPs. The new nanoparticles exhibit better anti-HIV effects compared to nanoparticles produced through the conventional Turkevich method. The novel nanoparticles produce virucidal and inhibitory effects by occupying attachment sites and preventing the virus from binding with T-lymphocyte, or modifying proteins involved in HIV replication.

Keywords: Human immunodeficiency virus, Gold nanoparticle, Rosa canina, Peripheral blood mononuclear cells
Volume 6 Issue 3 - 2017

\author{
Pooriya Khademi-Azandehi,' Javad \\ Moghaddam, ${ }^{2}$ Khademi-Azandehi Roozbeh, ${ }^{3}$ \\ Sayed-Saeed Veradi-Esfahani, ${ }^{4}$ Shahabi-Satlsar \\ Esmaeil $^{5}$ \\ 'Materials Engineering Department, Sahand University of \\ Technology, Iran \\ ${ }^{2}$ Department of Materials Science, University of Zanjan, Iran \\ ${ }^{3}$ Health Department, Kerman University of Medical Sciences and \\ Health Services, Iran \\ ${ }^{4}$ Biomedical Engineering Department, Sahand University of \\ Technology, Iran \\ ${ }^{5}$ Paramedical Department, Iran University of Medical Sciences, \\ Iran
}

Correspondence: Javad Moghaddam, Department of Materials Science, Faculty of Engineering, University of Zanjan, P.O. Box 4537I-3879I, Zanjan, Iran, Tel 98912 I4I 3049, Fax 98243305 4364 Email moghaddam@znu.ac.ir

Received: October 19, 2017 | Published: November 09, 2017
Abbreviations:HIV, Human Immunodeficiency Virus; PBMC, Peripheral Blood Mononuclear Cells; SPR, Surface Plasmon ResonanceAuNPs, Au (gold) Nanoparticles

\section{Introduction}

AuNPs have been in the focal point of researchers' attention for years because of high biocompatibility and adjustable surface plasmon resonance (SPR) . In the recent years, many studies have been performed in producing AuNPs via green synthesis techniques, i.e. use of plants for producing AuNPs.$^{2}$ Green synthesis methods are eco-friendly and increase the biocompatibility of AuNPs. ${ }^{3} \mathrm{~A}$ factor that gives rise to the highest difference between various gold nanoparticle characteristics is the size of synthesized AuNPs and their superficial compounds . ${ }^{4}$ Rosa canina fruit extract has immunomodulatory characteristics with probable potential to be used in treatment of immunodeficiency diseases . Rosa canina fruit extract, can reduce initial gold salt - chloroauric acid - and produce gold colloid. The major components of this plant are Vitispiran, 5-Methyl-3-hexanone, 2-Heptanone, Hexadecanoic and Dodecanoic acid, Linolic acid, $\alpha$-EAcaridial, Myristic acid, Benzaldehyde, $\alpha$-pinene, 2-pentylfuran, 2,4-Heptadienal, 1,4-Epoxy-p-menthane, Salicylaldehyde, 2-Heptenol and Linalool.$^{6}$ In this research, nanoparticles were produced via a novel green method and capped with Rosa canina fruit extract. Then, the antiretroviral effects of these nanoparticles were investigated. Size stability of AuNPs in physiological condition $(\mathrm{pH}=7.4)$ is the necessary condition for their application in medicine . ${ }^{7}$ The stability of AuNPs produced using Rosa canina fruit extract and nanoparticles produced through the conventional sodium citrate reducing agent in $\mathrm{pH}=7.4$ was thus compared. After the human immunodeficiency virus (HIV-1) enters the body and the blood circulatory system, its superficial glycoproteins, gp120 and gp41, bind with receptors like CD4 and CCR5 on the surface of T-Lymphocytes. By sending its capsid into T-lymphocytes, this virus inducts its RNA together with enzymes, such as reverse transcriptase, integrase and protease into the cell, allowing them to start an operation that will force the T-cells to produce new HIVs. Because of the high infection volume of this disease, antiretroviral drugs are employed to control its advance. Most of these drugs are transcriptase inhibitors.$^{8}$ integrase inhibitors.$^{9}$ and protease inhibitors.${ }^{10}$ although they cannot be completely effective, because of HIV's resistance to drugs. The purpose of this study was to use the new reducing agent - Rosa canina fruit extract- to produce nanoparticles with anti-HIV effects in order to decrease the number of viruses in the bloodstream. Ongoing reduction in the number of viruses in the blood, and consumption of antiretroviral drugs can rise the incubation period and postpone the outbreak of AIDS symptoms.

\section{Material and methods}

\section{Rosa canina fruit aqueous extract provision}

$10 \mathrm{~g}$ of Rosa canina fruit was boiled for 45 minutes in $200 \mathrm{ml}$ deionized water which was then filtered several times to obtain Rosa canina fruit extract. 


\section{Synthesis of AuNPs}

$2 \mathrm{ml}$ Rosa canina fruit extract was added to $25 \mathrm{ml}$ of $1 \mathrm{~m} \mathrm{M}$ chloroauric acid. The compound was stirred in $85^{\circ} \mathrm{C}$ for 5 minutes. The change in the mixture's color from bright yellow to wine red, indicated that nanoparticles have been produced.$^{11}$ Citrate-capped AuNPs were also produced through the Turkevich method.$^{12}$

\section{Gold nanoparticle spectroscopy via UV-visible and FT- IR Analyses}

UV-Shimadzu 1700 was utilized to obtain gold nanoparticle absorption spectrum in the range of 400 to $800 \mathrm{~nm}$. Bruker Tensor 27 was employed to obtain the FT-IR spectra.

\section{Investigation of size, dimensional stability,zetapotential and morphology of particles via DLS and TEM}

Dynamic light scattering (DLS) data was acquired using a Nanotrac wave device to obtain the size and zeta potential of synthesized nanoparticles. Stability of nanoparticles produced using Rosa canina fruit extract and citrate-capped nanoparticles was investigated in both the synthesis and the physiological conditions $(\mathrm{pH}$ =7.4). Moreover, stability of nanoparticles synthesized using Rosa canina fruit extract was studied in both time-variant and temperaturevariant tests. In the time-variant test, the stability of nanoparticles was studied for 20 days while in the temperature-variant test, dimensional stability was studied between $20^{\circ} \mathrm{C}$ and $45{ }^{\circ} \mathrm{C}$. Furthermore, the size and morphology of nanoparticles was obtained via transmission electron microscopy (TEM) using a $100 \mathrm{kV}$ LEO906.

\section{Cytotoxicity evaluation of nanoparticles via MTT assay}

MTT assay can use Vero cell line to obtain the optimal concentration of AuNPs before using them in antiviral applications .${ }^{13}$ As the mitochondrial cellular enzymes can reduce tetrazolium to formazan crystals, cells were cultured in 96 cell culture well plate. It was then kept in an incubator for 24 hours in 5\% carbon dioxide at 37 ${ }^{\circ} \mathrm{C}$. Different concentrations of nanoparticles were then added to wells and the plate was incubated again for 10 hours in $\mathrm{CO} 2$ gas atmosphere at $37{ }^{\circ} \mathrm{C}$. Then, $20 \mu \mathrm{MTT}$ reagent with $5 \mathrm{mg} / \mathrm{ml}$ was added to each well and the plate was placed in a $\mathrm{CO} 2$ incubator for 4 hours at 37 ${ }^{\circ} \mathrm{C}$. After taking the plate out of the incubator, $100 \mu$ of the dimethyl sulfoxide (DMSO) was added to each well. In the final step, after placing the plate on shaker for 5 minutes, the amount of absorption by the ELISA Reader device in $540 \mathrm{~nm}$ was obtained.

\section{Separating PBMCs of healthy and HIV-infected donors and enhancing T-lymphocyte replication}

After the sampling, $5 \mathrm{ml}$ of the donor's blood was combined with $5 \mathrm{ml}$ of phosphate-buffered saline (PBS) solution and homogenized. Then, the obtained mixture was slowly added to $3 \mathrm{ml}$ of Ficoll-1077 in a way that the blood and PBS came to the top of Ficoll. The mixture vessel was centrifuged under $400 \mathrm{G}$ acceleration for 20 minutes. Then, separation of PBMCs from the plasma layer and Ficoll was done by means of a pipette. The separated PBMCs were washed twice by adding PBS and centrifuging under $100 \mathrm{G}$ acceleration for 10 minutes. RPMI growth medium and Fetal Bovine Serum (FBS) were added to the PBMCs, which were kept in $\mathrm{CO}_{2}$ incubator for one day with $37{ }^{\circ} \mathrm{C}$ and kept in T25 flask. After 24 hours of incubation, PBMCs were again centrifuged under $100 \mathrm{G}$ for 10 minutes and added with RPMI growth medium, FBS, mitogen phytohemagglutinin (PHA) and Human interleukin 2 (IL-2). The mixture was kept in $\mathrm{CO}_{2}$ incubator for 72 hours and $37^{\circ} \mathrm{C}$. PHA increases the replication rate of PBMCs and IL-2 causes excessive replication of T-lymphocytes ..$^{14}$

\section{Preparing virus supply and tissue culture infective dose $50 \%$ (TCID50)}

PBMCs stimulated by PHA and IL-2 were centrifuged and the supernatant was discarded. Then, the PBMCs separated from HIVpositive donor was combined in 2:1 volumetric ratio and kept in $\mathrm{CO}_{2}$ incubator for 30 days in $37^{\circ} \mathrm{C}$. Meanwhile once every three days, the supernatant was separated such that the PBMCs in the flask would not be harmed. The removed mass was replaced by the same amount of a mixture of the warm growth medium and PBMCs stimulated by PHA. The culture supernatant separated from the flask, which contains a virus supply, was reserved in $-70{ }^{\circ} \mathrm{C}$. This three day cycle was repeated for 30 days. After preparing the virus supply, TCID50 must be calculated according to relevant Protocols.$^{15}$ for further experiments. TCID50 shows the amount of virus that is able to infect $50 \%$ of the healthy cultured cells. For this purpose, PBMCs stimulated with PHA were centrifuged; the supernatant was then discarded and the stimulated PBMCs were suspended in the growth medium. In this step, the cells were counted by means of trypan blue. When at least $85 \%$ of cells were verified to be alive, the growth medium, together with the mentioned PBMCs was added to 96 cell culture well plate, and all the wells were added with $200 \mu$ of PBS. Then, the ice crystal from the reserved virus supply was warmed up in a 27 ${ }^{\circ} \mathrm{C}$ water bath. The virus supply was immediately added with growth medium twelve times its volume. In this test, seven columns of wells in three rows, were exposed to the virus supply, such that $200 \mu \mathrm{l}$ of virus supply was added to three first rows of the first column and then, by means of a multichannel micropipette, $50 \mu 1$ of the first infected well column was transferred to the next column. The same procedure was followed for the next columns while the micropipette needle was changed after each transfer from one column to the next. $50 \mathrm{ml}$ of the seventh column wells were also discarded. Then, the plate was kept in $\mathrm{CO}_{2}$ incubator for four days. After discarding $125 \mu \mathrm{l}$ of each well's cellular suspension, growth medium was again added to the wells and incubation was performed for three more days. In the seventh day, p24 antigen test was performed to calculate TCID50. The p24 antigen test assigned positive or negative signs to the wells containing virus supply. In the end, TCID50 was calculated via Spearman-Karber method.$^{16}$

$$
M=x k+d\left[0.5-\left(\frac{1}{n}\right) r\right]
$$

Where $x k$ is the highest dilution dose, $d$ is the spacing between dilutions, $r$ is the sum of the number of negative responses and $n$ is the wells per dilution.$^{17}$

\section{Targeting HIV and HIV infection by AuNPs}

In order to study the effect of AuNPs on precluding HIV infection, the tests were designed to allow the effect of nanoparticles on controlling virus replication, both before and after the infection of T-lymphocytes to be investigated. Therefore three types of tests were performed:

a. The viruses were only subjected to interaction with PBMCs stimulated by PHA

b. The viruses were first subjected to interaction with PBMCs stimulated by PHA for two hours and then their mixture was exposed to interaction with AuNPs (secondary interaction)

c. The viruses were first exposed to interaction with AuNPs for two hours and then their mixture was subjected to interaction with PBMCs stimulated by PHA (initial interaction) 
In all three types of tests, the virus suspension volume, as well as nanoparticle volume added to each well was $100 \mu$ l. In these tests, 5 ppm concentration of nanoparticles was utilized, because a negligible cytotoxicity of particles have been evaluated in this concentration, according to MTT assay results. These three tests were performed by citrate-capped AuNPs as well as nanoparticles capped with Rosa canina fruit extract. The outcome of each of the three tests was subjected to p24 antigen antiviral susceptibility test (ELISA). The p24 antigen test (ELISA) is based on the connection of antibodies possessing color generator substances to $\mathrm{p} 24$ antigen. This test was performed under relevant standards.$^{18}$ High levels of p24 antigen indicate high infection. To investigate the performance of AuNPs in preventing virus replication, p24 test (ELISA) results were compared between the test in which only viruses and PBMCs were present and the tests including nanoparticles.

\section{Results}

\section{Synthesis of Nanoparticles and Their Characterization}

Two types of AuNPs produced by precipitation method. The reducing agents for novel AuNPs and conventional AuNPs were Rosa canina fruit extract and sodium citrate respectively. Both nanoparticles were subjected to spectroscopy tests to obtain the information about chemical bonds on their surface. The dimensional stability of AuNPs was investigated in different times and temperatures via DLS analysis. Also, the mostly spherical morphology of AuNPs was obtained via TEM. The particle size in the TEM image, confirms the data from DLS analysis. The stability survey tests showed that novel particles are more stable in the biological temperature range. Figure 1 shows the UV-visible spectrum of AuNPs produced using Rosa canina fruit extract in synthesis as well as physiological conditions. The SPR of nanoparticles showed no significant change in transition from synthesis to physiological condition and was $523 \mathrm{~nm}$ and $530 \mathrm{~nm}$ respectively.

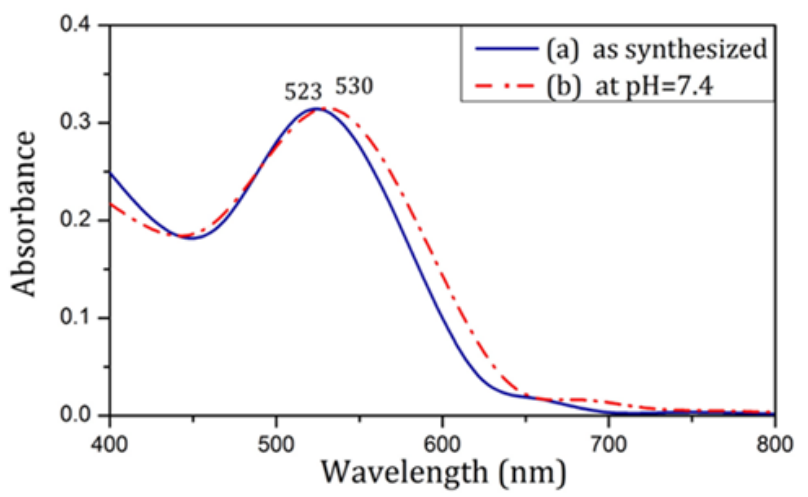

Figure I Absorption spectra of nanoparticles produced using Rosa canina fruit extract as synthesized (a) and in $\mathrm{pH}=7.4$ (b).

Figure 2 shows the UV-visible spectrum of nanoparticles produced by the use of sodium citrate in both synthesis and physiological conditions. The SPR of nanoparticles was drastically different across the two sets of condition, such that in synthesis condition, the SPR was significantly less than the SPR in physiological condition.

Table 1 shows the zeta potential of nanoparticles produced using sodium citrate and Rosa canina fruit extract in both synthesis and physiological conditions. According to Table 1, severe change in zeta potential belongs to nanoparticles produced using sodium citrate. Figure 3 shows the size distribution of AuNPs synthesized using Rosa canina fruit extract. As shown in this figure, the size of these nanoparticles was under $100 \mathrm{~nm}$ and the average size was $27.7 \mathrm{~nm}$. Also most nanoparticles were concentrated around $20 \mathrm{~nm}$ and $50 \mathrm{~nm}$.

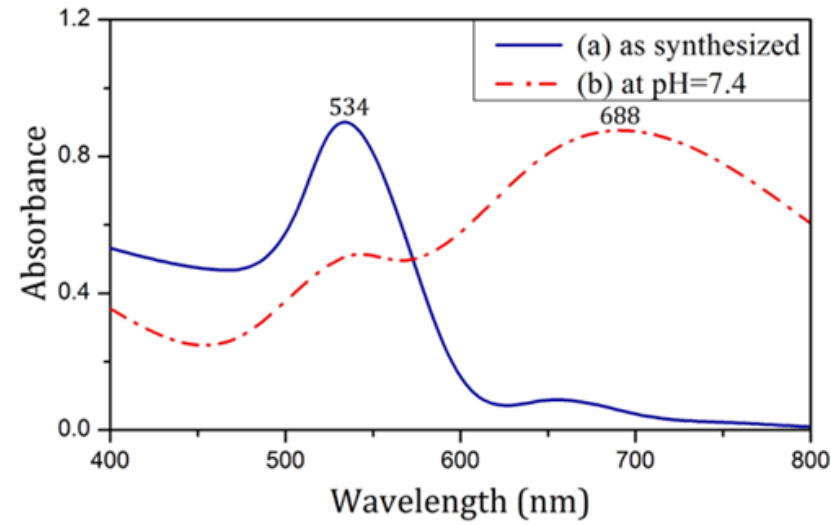

Figure 2 Absorption spectra of nanoparticles produced using Sodium citrate as synthesized (a) and in $\mathrm{pH}=7.4$ (b).

Table I Zeta potential of gold nanoparticles synthesized using sodium citrate and Rosa canina fruit extract.

\section{Zeta Potential (mV)}

\begin{tabular}{lll} 
& As Synthesized & At pH $=7.4$ \\
\hline $\begin{array}{l}\text { Nanoparticles synthesized using citrate } \\
\text { Nanoparticles synthesized using Rosa }\end{array}$ & $-39 \pm 2.10$ & $-\mathrm{II} \pm \mathrm{I} .50$ \\
$\begin{array}{l}\text { canina fruit } \\
\text { and }\end{array}$ & $+36 \pm \mathrm{I} .30$ & $+34 \pm \mathrm{I} .40$
\end{tabular}

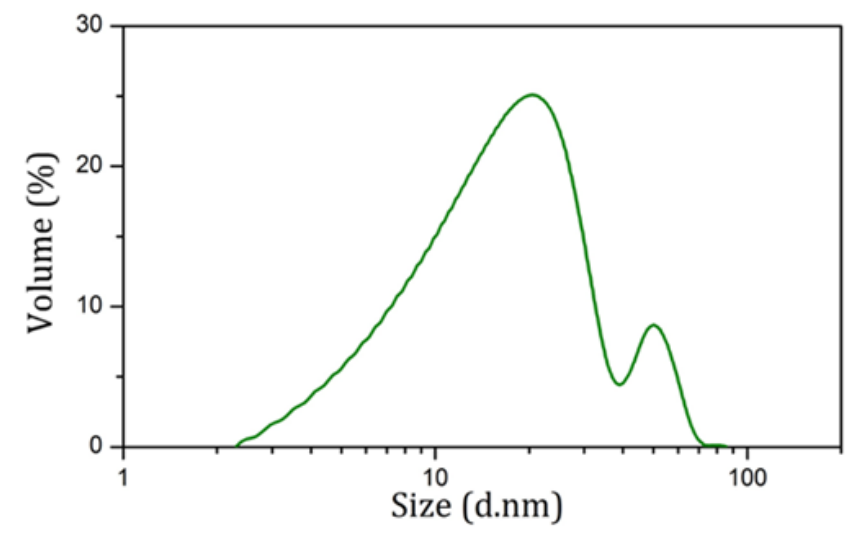

Figure 3 Size distribution diagram of AuNPs produced using Rosa canina fruit extract obtained via dynamic light scattering.

Figure 4 shows the FT-IR spectra of nanoparticles synthesized using Rosa canina fruit extract. Three bands are clearly visible in $1641 \mathrm{~cm}^{-1}, 2000 \mathrm{~cm}^{-1}$, and $3477 \mathrm{~cm}^{-1}$. The bands located in $1641 \mathrm{~cm}$ ${ }^{1}$ and $2000 \mathrm{~cm}-1$ may belong to stretching vibration of $\mathrm{C}=\mathrm{C}$ and overtones respectively.$^{19,20}$ Also, the band occurring in $3447 \mathrm{~cm}^{-1}$ may be assigned to the stretching vibrations of $\mathrm{O}-\mathrm{H} .{ }^{21}$ Figures 4 \& 6 shows the stability of nanoparticles produced using Rosa canina fruit extract in 20 days and in biological temperature range respectively, via DLS technique. Figure 7 shows a TEM image of nanoparticles produced using Rosa canina fruit extract. Most nanoparticles exhibit dimensions under $30 \mathrm{~nm}$ and cause the mixture to be monodisperse.

\section{Cytotoxicity evaluation of nanoparticles via MTT assay}

Estimation of AuNPs cytotoxicity is highly important in medical applications. During MTT assay, mitochondrial enzymes of the cells reduce tetrazolium to blue-violet formazan crystals. If nanoparticle cytotoxicity is higher, the amount of mitochondrial enzymes decreases, consequently that will affect the final color of the mixture 
in the plate. Figure 8 displays the MTT assay results for both types of synthesized nanoparticles. The assay was performed in three different concentrations of nanoparticles. The proper concentration for performing biological experiments was $5 \mathrm{ppm}$ which had the least cytotoxicity.

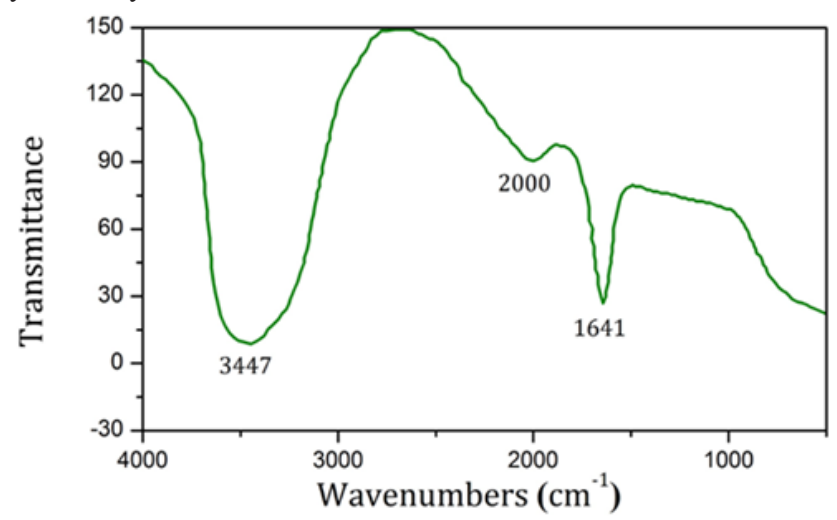

Figure 4 FTIR spectrum of AuNPs synthesized using Rosa canina fruit extract.

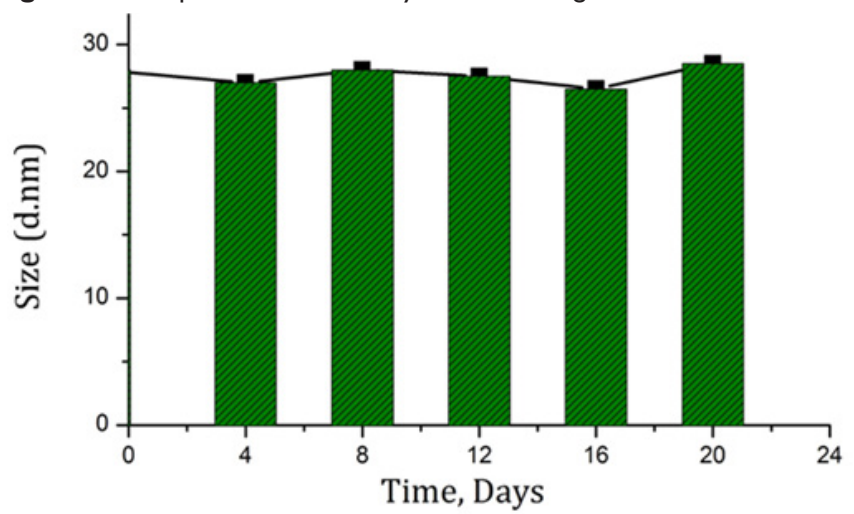

Figure 5 Average size of AuNPs produced using Rosa canina fruit extract in $\mathrm{pH}=7.4$ over time.

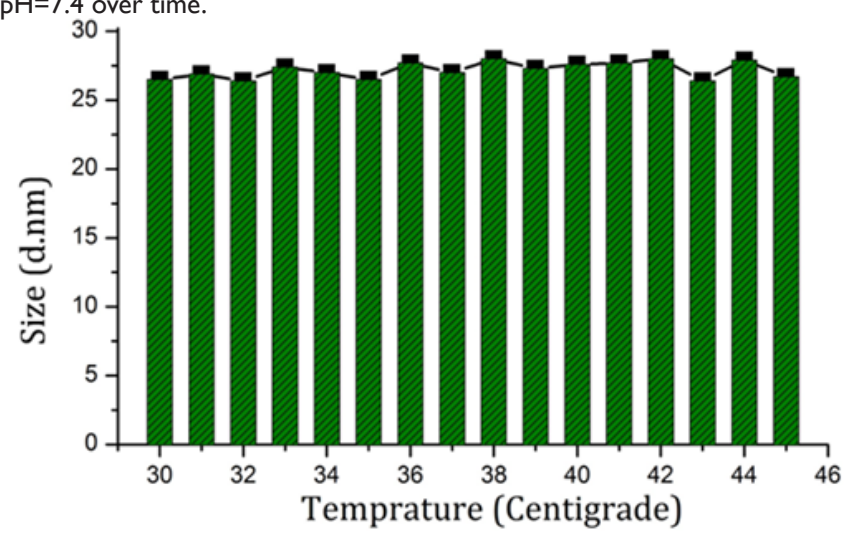

Figure 6 Average size of AuNPs synthesized using Rosa canina fruit extract in $\mathrm{pH}=7.4$ in and around biological temperature.

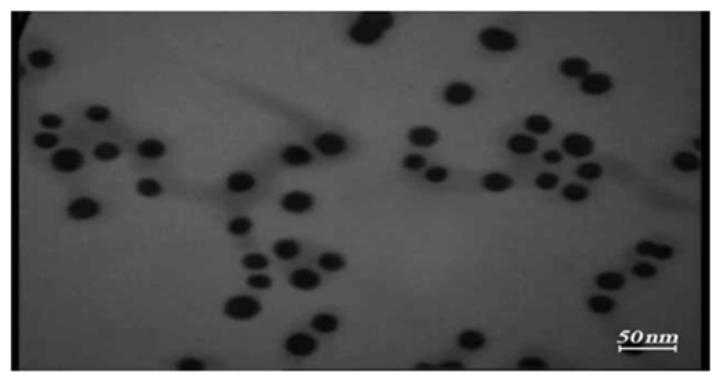

Figure 7 TEM image of AuNPs produced using Rosa canina fruit extract.

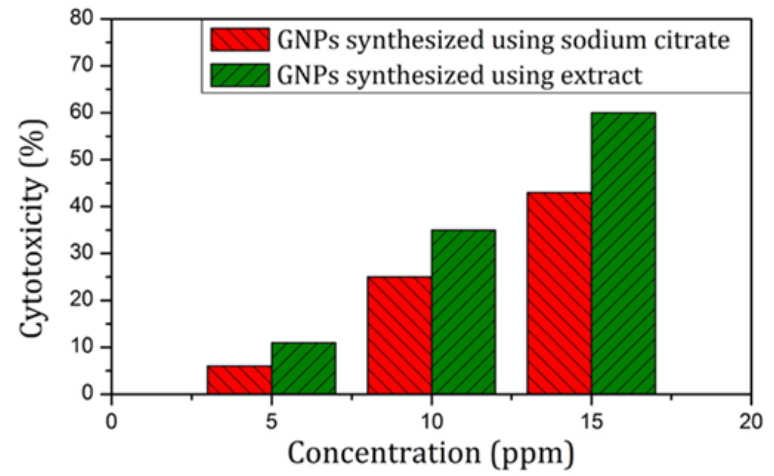

Figure 8 Cytotoxicity percentage in various concentrations of both types of AuNPs.

\section{HIVTargeting}

Both types of nanoparticles were separately mixed with the viruses and the resulting mixture was interacted with PBMCs (initial interaction). Furthermore, the viruses were mixed with PBMCs, and the resulting mixture was subjected to interaction with both types of nanoparticles separately (secondary interaction). Figure 9 shows the percent inhibition of p24 antigen in different types of tests involving interaction between AuNPs and the viruses. Maximum infection occurs when PBMCs are exposed to HIVs in the absence of AuNPs. In this type of test, the percent inhibition of p24 antigen was considered zero and the respective infection was considered $100 \%$. These assumptions was used to obtain the percent inhibition of p24 antigen for the types of test in which HIVs were exposed to AuNPs, both before the infection of PBMCs (initial interaction) and afterwards (secondary interaction). According to Figure 9, the highest the percent inhibition of p24 antigen corresponds to nanoparticles produced using Rosa canina fruit extract, when nanoparticles interacted with the viruses first and HIVs infected the PBMCs later.

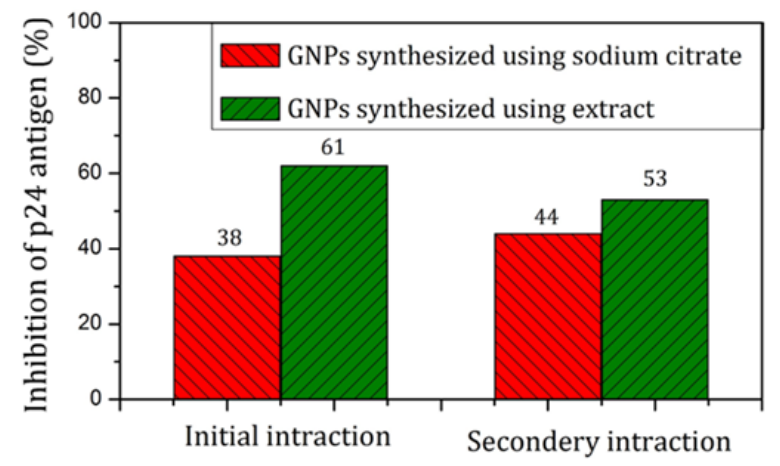

Figure 9 Percent inhibition of p24 antigen when exposed to interaction with both types of nanoparticles, before and after PBMCs are infected by HIVs.

\section{Discussion}

As shown in Figure 1, nanoparticles synthesized using Rosa canina fruit extract had a SPR band at $523 \mathrm{~nm}$. No significant difference was observed in the particle SPR under physiological condition $(\mathrm{pH}$ = 7.4). To provide physiological condition, isotonic PBS solution, which contains the same amount of ions and has the same $\mathrm{pH}$ as human blood was utilized. According to Mie theory, SPR invariance in $\mathrm{pH}=7.4$ signifies AuNP size stability in physiological condition.$^{22}$ As shown in Figure 2, the SPR of nanoparticles produced with sodium citrate in synthesis condition was $534 \mathrm{~nm}$. After being placed in PBS solution $(\mathrm{pH}=7.4)$, the SPR band was broadened and its peak moved 
towards longer wavelengths, which indicates aggregation of AuNPs according to Mie theory ${ }^{23}$ The change in the SPR of citrate-capped AuNP can be analyzed through zeta sizer data. The results in Table 1 show that applying physiological condition drastically decreased the zeta potential absolute value of citrate-capped nanoparticles. PBS solution ions interacted with superficial nanoparticle ions and caused the surface electrical charge of nanoparticles to decrease, which in turn reduced the electrostatic repulsion between them, causing them to attach and aggregate.$^{24}$ According to Table 1 , in contrast with citrate-capped nanoparticles, the zeta potential of nanoparticles produced using Rosa canina fruit extract, did not change significantly, under physiological condition. The issue can be explained in terms of compatibility between PBS solution ions and the surface charge of nanoparticles. In other words, the surface electrical charge of a large portion of PBS solution ions was the same as surface electrical charge of particles, which avoids the decrease of electrostatic repulsion of the nanoparticles.

According to previous researches, among the various compounds that comprise plants, flavonoids possess better reducing abilities ${ }^{25}$ Therefore, these compounds are probably responsible for the reduction of chloroauric acid and production of nanoparticles. $\mathrm{C}=\mathrm{C}$, $\mathrm{O}-\mathrm{H}$ and overtones bonds visible in Figure 4, indicate the presence of a portion of extract compounds on nanoparticles' surface, which is the cause for their dimensional stability. These bonds can be found in terpenoids, fatty acids, aromatic aldehydes and some chelates . Figure 5 shows the size stability of nanoparticles synthesized using Rosa canina fruit extract during 20 days. The results show that during this time, mean nanoparticle size has not changed much. Moreover, Figure 6 displays their dimensional stability in the biological temperature range, i.e. human body temperature. The results suggest that the coat covering nanoparticles synthesized using Rosa canina fruit extract has the ability to avoid aggregation of nanoparticles over time and with the rise of temperature. Nanoparticles with spherical morphology are also visible in the TEM image. Nanoparticle size observable in this image is in clear concordance with DLS analysis results. In general, dimensional stability in a medium resembling human blood, in $\mathrm{pH}=7.4$, in biological temperature and over time, cause nanoparticles produced using Rosa canina fruit extract to have the necessary conditions for application in medicine.$^{7}$ In targeting HIV and HIV infection, two types of interaction were investigated. In the initial interaction, nanoparticles affect the virus before PBMCs were infected. In the secondary interaction, PBMCs were infected first and nanoparticles intervene later. The initial interaction is a criterion for targeting HIV while the secondary interaction is a measure for targeting HIV infection. As visible in Figure 9, percent inhibition of p24 antigen related to nanoparticles capped with citrate in both initial and secondary - interactions is less than that of nanoparticles synthesized using Rosa canina fruit extract. Therefore it can be suggested that nanoparticles produced using Rosa canina fruit extract perform stronger in targeting HIV and its infection than nanoparticles capped with sodium citrate. The reason for this variation, is the difference in compounds attached to the surface of AuNPs. Because the morphology of nanoparticles produced using sodium citrate and Rosa canina fruit extract are similar to each other, the difference between antiviral properties of these nanoparticles can be attributed to the compounds on their surface.${ }^{26}$ Recently, several studies have investigated the impact of noble metallic nanoparticles on HIV replication. Although variations in gp120, is the basis for difference among HIV strains, no significant difference have been reported between antivirus effect of nanoparticles on drug-resistant HIV strains.$^{27,28}$ Beside, viral absorption assays indicated that the dominant mechanism of anti-HIV activity of nanoparticles is inhibition of the virus replication cycle in its early stages. Inhibition of p24 antigen during initial interaction, shows the virucidal property of AuNPs. According to Figure 9, virucidal effect for nanoparticles capped with Rosa canina fruit extract is stronger than sodium citrate capped nanoparticles. The first step in viral replication, is the attachment of gp120 to CD4 receptor which is an electrostatic interaction. Positive charge nanoparticles can interact with negative cavity of gp120 and prevent gp120 from binding with CD4.$^{29}$ As nanoparticles capped with Rosa canina fruit extract are positively charged, they are more successful in binding with gp120 than sodium citrate capped nanoparticles which are negatively charged. These nanoparticles interact with gp120 and prevent it from binding with CD4. Therefore, in accordance with Figure 9, The superiority of virucidal properties of nanoparticles capped with Rosa canina fruit extract compared to that of citrate capped AuNPs, can be attributed to the positive charges of their surface. Moreover, the gold ions in a AuNP solution can bind with sulfhydryl groups and reduce the disulfide bonds in the carboxyl half of gp120. Disulfide bonds are among the areas involved in binding to CD4 receptor.$^{30,31}$ Reduction of the disulfide bonds in a protein, causes the protein to denaturate. The denaturation is an irreversible process which leads to viral structure modification.$^{32,33}$ Therefore, in addition to preventing interaction between gp120 and CD4, AuNPs change the characteristics of superficial glycoproteins of the virus. It seems that AuNPs attach to gp120 first, and modify viral proteins next; blocking viral entry and creating virucidal effect. Due to the positive charge on the surface of the nanoparticles produced using Rosa canina fruit extract, these AuNPs are more likely to bind with gp120, compared to citrate capped nanoparticles. Thus, they can perform better in viral protein modification and display a stronger virucidal effect. In addition, AuNPs may affect the later stages of the virus life cycle. As shown in Figure 9 AuNPs display inhibitory effect on the replication of viruses placed inside PBMCs as well. According to prior research, nanoparticles can increase the permeability of the cell membrane and enter the cytoplasm.$^{34,35}$ AuNPs use this mechanism to enter infected PBMCs. Electron donor groups such as amino acids, thiols, phosphates and nucleic acids containing elements such as nitrogen, oxygen and sulfur, are able to complex with gold ions and block proteins involved in HIV replication. This can decrease reverse transcription or transcription rates.$^{36}$ As can be seen in Figure 9, p24 antigen inhibition in PBMCs is more prevalent by nanoparticles covered with Rosa canina fruit extract compared to nanoparticles coated with sodium citrate. Perhaps it can be argued that the active components in Rosa canina fruit extracts such as terpenoids, modify the structure of these proteins by binding with proteins which enter the PBMCs and disrupt the function of at least one of such enzymes as reverse transcriptase, integrase or protease; or decrease transcription by binding with DNA and RNA molecules. Further information in line with confirming in vitro results, requires in vivo clinical experiments.

\section{Conclusion}

This research showed that Rosa canina fruit extract can be used as a new powerful reducing and stabilizing agent for synthesizing AuNPs. In contrast with nanoparticles produced using sodium citrate, nanoparticles produced in this method had the necessary conditions for application in medicine, i.e. they were not aggregated in a medium with bloodlike ionic concentration, in $\mathrm{pH}=7.4$, in biological temperature and overtime. They also displayed anti-HIV characteristics, which was visible both before and after infection by the virus. The anti-HIV characteristics of nanoparticles synthesized using Rosa canina fruit extract was more sensible than nanoparticles produced through the conventional Turkevich method. 


\section{Acknowledgements}

Our special thanks goes to Khazar Environmental Health Services Company, who funded this non-profit research in good faith.

\section{Conflicts of Interest}

The authors declare no conflict of interest.

\section{References}

1. Zhang P, Li B, Du J, Wang Y Regulation the morphology of cationized AuNPs for effective gene delivery. Colloids Surf B Biointerfaces. 2017; 157:18-25.

2. Santhoshkumar J, Rajeshkumar S, Kumar SV Phyto-assisted synthesis, characterization and applications of AuNPs-A review. Biochemistry and Biophysics Reports. 2017;11:46-57.

3. Ahmad N, Bhatnagar S, Saxena R et al. Biosynthesis and characterization of AuNPs: Kinetics, in vitro and in vivo study. Mater Sci Eng C Mater Biol Appl. 2017;78:553-564.

4. Cardoso Avila PE, Rangel Mendoza A, Pichardo Molina JL et al. Biological response of $\mathrm{HeLa}$ cells to AuNPs coated with organic molecules. Toxicol In Vitro. 2017;42:114-122.

5. Sadigh-Eteghad S, Tayefi-Nasrabadi H, Aghdam Z et al. Rosa canina L. fruit hydro-alcoholic extract effects on some immunological and biochemical parameters in rats. Bioimpacts. 2011;1(4):219.

6. Nowak R Chemical Composition of Hips Essential Oils of Some Rosa L. Species. Z Naturforsch C. 2005;60(5-6):369-378.

7. Ajdari N, Vyas C, Bogan SL et al. Gold nanoparticle interactions in human blood: a model evaluation. Nanomedicine: Nanotechnology, Biology and Medicine. 2017;13(4):1531-1542.

8. Agarwal HK, Chhikara BS, Doncel GF et al. Synthesis and antiHIV activities of unsymmetrical long chain dicarboxylate esters of dinucleoside reverse transcriptase inhibitors. Bioorg Med Chem Lett. 2017;27(9):1934-1937.

9. Barber $\mathrm{T}$ Integrase inhibitors go head-to-head. Lancet HIV. 2017;4(4):142e-143e.

10. Ghosh AK, Sarkar A An enantioselective enzymatic desymmetrization route to hexahydro-4H-furopyranol, a high-affinity ligand for HIV-1 protease inhibitors. Tetrahedron Letters. 2017;58(33):3230-3233.

11. Stolarczyk EU, Stolarczyk K, Łaszcz M et al. Pemetrexed conjugated with AuNPs-Synthesis, characterization and a study of noncovalent interactions. Eur J Pharm Sci. 2017;109:13-20.

12. Shi L, Buhler E, Boué F, Carn F How does the size of AuNPs depend on citrate to gold ratio in Turkevich synthesis? Final answer to a debated question. J Colloid Interface Sci. 2017;492:191-198.

13. Houdkova M, Rondevaldova J, Doskocil I et al. Evaluation of antibacterial potential and toxicity of plant volatile compounds using new broth microdilution volatilization method and modified MTT assay. Fitoterapia. 2017;118:56-62.

14. Małaczewska J, Wójcik R, Kaczorek E et al. Commercial gold nanocolloid inhibits synthesis of IL-2 and proliferation of porcine T lymphocytes. Res Vet Sci. 2017;110:4-11.

15. LaBarre DD, Lowy RJ Improvements in methods for calculating virus titer estimates from TCID 50 and plaque assays. J Virol Methods. 2001;96(2):107-126.

16. Muthannan Andavar Ramakrishnan Determination of $50 \%$ endpoint titer using a simple formula. World J Virol. 2016;5(2):85-86.

17. ACTG Laboratory Technologist Committee, TCID50 Determination of Viable HIV-1, ACTG Lab Man. 2004;1: 1-10.
18. Benmarzouk-Hidalgo OJ, Torres-Cornejo A, Gutierrez-Valencia A et al. HIV-1 p24 and CD4+ T cell count during boosted protease-inhibitor monotherapy in HIV-infected patients. Enfermedades infecciosas $y$ microbiologia clinica. 2017;35(3):174-178.

19. Philip D Honey mediated green synthesis of AuNPs. Spectrochim Acta A Mol Biomol Spectrosc. 2009;73(4):650-653.

20. Alvarez-Ordóñez A, Prieto M Fourier transform infrared spectroscopy in food microbiology. Springer, Germany. 2012

21. Khademi-Azandehi P, Moghaddam J Green synthesis, characterization and physiological stability of AuNPs from Stachys lavandulifolia Vahl extract. Particuology. 2015;19:22-26.

22. Lysak VV Optical properties of core/shell nanoparticles: Comparison of $\mathrm{TiO} 2 / \mathrm{Ag}$ and $\mathrm{Ag} / \mathrm{TiO} 2$ structures. Materials Today: Proceedings. 2017;4(3):4890-4895.

23. Ladutenko K, Pal U, Rivera A et al. Mie calculation of electromagnetic near-field for a multilayered sphere. Computer Physics Communications. 2017;214:225-230.

24. Manson J, Kumar D, Meenan BJ et al. Polyethylene glycol functionalized AuNPs: the influence of capping density on stability in various media. Gold bull. 2011;44(2):99-105.

25. Irfan M, Moniruzzaman M, Ahmad T et al. Ionic liquid based extraction of flavonoids from Elaeis guineensis leaves and their applications for AuNPs synthesis. Journal of Molecular Liquids. 2017;241:270-278.

26. Di Gianvincenzo P, Marradi M, Martínez-Avila OM et al. AuNPs capped with sulfate-ended ligands as anti-HIV agents. Bioorg Med Chem Lett. 2010;20(9):2718-2721.

27. Neurath AR, Strick N, Li YY, Debnath AK Cellulose acetate phthalate, a common pharmaceutical excipient, inactivates HIV-1 and blocks the coreceptor binding site on the virus envelope glycoprotein gp120. BMC infectious diseases. 2001;1(1):17.

28. Spira S, Wainberg MA, Loemba H et al. Impact of clade diversity on HIV-1 virulence, antiretroviral drug sensitivity and drug resistance. $J$ Antimicrob Chemother. 2003;51(2):229-240.

29. Kwong PD, Wyatt R, Robinson J et al. Structure of an HIV gp120 envelope glycoprotein in complex with the CD4 receptor and a neutralizing human antibody. Nature. 1998;393(6686):648-659.

30. Lekutis C, Olshevsky U, Furman C et al. Contribution of disulfide bonds in the carboxyl terminus of the human immunodeficiency virus type I gp120 glycoprotein to CD4 binding. J Acquir Immune Defic Syndr. 1991;5(1):78-81.

31. Schaefer K, Webb NE, Pang M, Hernandez-Davies JE, Lee KP, et al. (2017) Galectin-9 binds to $\mathrm{O}-$ glycans on protein disulfide isomerase. Glycobiology. 1991;27(9):878-887.

32. Lara HH, Ayala-Nuñez NV, Ixtepan-Turrent L et al. Mode of antiviral action of silver nanoparticles against HIV-1. J Nanobiotechnology. 2010;8(1):1.

33. Manteca A, Alonso-Caballero Á, Fertin $M$ et al. The Influence of Disulfide Bonds on the Mechanical Stability of Proteins is Context Dependent. J Biol Chem. 2017;292(32):13374-13380.

34. Xu XH, Brownlow WJ, Kyriacou SV et al. Real-time probing of membrane transport in living microbial cells using single nanoparticle optics and living cell imaging. Biochemistry. 2004;43(32):10400-10413.

35. Falanga AP, Pitingolo G, Celentano $M$ et al. Shuttle-mediated nanoparticle transport across an in vitro brain endothelium under flow conditions. Biotechnology and bioengineering .2017;114(5): 1087-1095.

36. Starodub ME, Trevors JT Silver resistance in Escherichia coli R1. J Med Microbiol. 1989;29(2):101-110. 